\title{
Peijian Chen · Shaohua Chen \\ Contact behaviors of a rigid punch and a homogeneous half-space coated with a graded layer
}

Received: 2 August 2011 / Revised: 26 October 2011 / Published online: 2 December 2011

(C) Springer-Verlag 2011

\begin{abstract}
A contact model between a homogeneous half-space with a linearly graded layer and a rigid punch is proposed and studied in the present paper. The governing equation, which describes the relation of the displacements and the normal tractions at the contact interface, is obtained by means of Fourier transform and a transfer matrix method. Appropriate collocation methods are used in order to solve the equation numerically. Singular behaviors at the edge of a flat punch are revealed. Compared to the case with a graded surface varying according to an exponential law, stress concentration is relatively weaker in the case with the graded surface varying according to a linear law. Furthermore, stress distributions in cases of a flat or cylindrical punch are given for different varying graded laws, thickness of graded layer, ratios of stiffness, and frictional coefficients. All the results are helpful for the design of strong and wear resistance coating surfaces.
\end{abstract}

\section{Introduction}

It is well known that frictional contact on homogeneously brittle substrates may give rise to special cracking patterns. Such cracks result from highly concentrated tensile stresses and are responsible for initiating failures related to sliding contact, as well as contact fatigue [1,2]. The applications of graded coating on metallic substrates provide a good solution to this problem. Used as coatings and interfacial zones, they tend to reduce stresses resulting from material property mismatch, increase the bonding strength, improve the surface properties, and provide protection against adverse thermal and chemical environment [3-7]. Graded materials have been chosen for many advanced engineering applications due to the unique mechanical and physical properties, such as bearings, gears, machine tools, cams, and abradable seals in gas turbines [8,9]. An important problem is how to design the graded layer in order to reduce the likelihood of cracking and to own excellent material toughness and wear resistance [10-17].

In the past few years, many studies indicate that controlling the gradient variation law in mechanical properties could offer unprecedented opportunities for surfaces to achieve improved wear resistance $[10,11,18,19]$. Giannakopoulos and Suresh $[20,21]$ studied an axisymmetric problem of a graded half-space subjected to a concentrated load or a flat, spherical or conical indenter. The modulus of the half-space is assumed to vary according to a simple power law $E(z)=E_{0} z^{k},(0 \leq k<1)$ or an exponential one $E(z)=E_{0} \mathrm{e}^{\alpha z}$ in depth. Significant changes of stresses around the stamp can be observed by adjusting the gradient variation of the elastic modulus. In 1999, Suresh et al. [18] carried out a numerical simulation and experimental investigation on a graded medium loaded by a sliding spherical indenter, in which the modulus of the medium is assumed to vary with the depth according to a power law, $E(z)=E_{s}+E_{0} z^{k}$, where $E_{s}$ is the surface value,

P. Chen $\cdot$ S. Chen $(\varangle)$

LNM, Institute of Mechanics, Chinese Academy of Sciences, Beijing 100190, China

E-mail: chenshaohua72@hotmail.com

Tel.: +86-10-82543960

Fax: +86-10-82543977 
$0<k<1$, and $E_{0}$ is selected so that the units are consistent. It is found that the controlled gradients in the elastic modulus alone can result in a pronounced enhancement in the resistance of a surface to frictional sliding contact. Chen et al. [22] discussed the adhesive contact problem of a rigid sphere in contact with a graded elastic half-space, in which Young's modulus of the half-space is varying with depth according to a power law $E(z)=E_{0}\left(z / c_{0}\right)^{k},(0<k<1)$. The corresponding two-dimensional problem was also investigated by Chen et al. [23] and Chen and Chen [24] later.

Among related papers, one of the early attempts is concerned with the contact problem of a rigid punch with a non-homogeneous half-plane. With the evolution of graded materials being extended to a coating layer with finite thickness, notable contributions have been made by Erdogan and his co-workers [3,8,9,25-27]. Guler and Erdogan [9] proposed a model that the shear modulus of the coating varies with depth according to an exponential law $\mu(y)=\mu_{2} \mathrm{e}^{\delta y},\left(0 \leq y \leq h, \delta=\log \left(\mu_{1} / \mu_{2}\right) / h\right)$, where $\mu(y), \mu_{2}$ are the shear modulus of the coating and substrate, respectively, $\delta$ is a constant characterizing the material inhomogeneity, and $h$ denotes the thickness of the coating. The plane strain contact problem for two deformable solids with FGM coatings was considered by Guler and Erdogan [8] in 2006. Ke and Wang [1,28] and Yang and Ke [29] proposed multi-layered models to analyze the preceding two problems and found significantly improved properties of FGM coatings in contact with rigid punches.

The problem in the present paper is inspired by the work done by Giannakopoulos and Pallot [30], in which a plane strain contact problem for a rigid stamp acting on a graded half-space is considered, and the Young's modulus of the semi-infinite graded medium is assumed to be $E(z)=E_{0} z^{k},(0 \leq k<1)$. They found that the distribution of contact pressure becomes more uniform when the elastic modulus of the semi-infinite graded medium gradually approaches a linear variation law in cases with both flat and cylindrical stamps. Is it true for the case of a finite coating? How are the stress intensity factors and distributions of contact stresses affected by the variation law of modulus, thickness of the graded layer, frictional coefficient, etc.? In order to answer the above questions, we established a contact model of a rigid punch in contact with a layered half-space in the present paper, in which the shear modulus of the graded layer varies according to a linear law. The linearly graded model captures the essential nature of the elastic modulus variation and is physically acceptable. We first obtained the relation of the surface displacement and the external concentrated force with the help of a model of a layer half-space acting by a concentrated force. Then, using integral methods, we could find the governing equation describing the relation of the interfacial displacements and the interfacial tractions in the contact model. Appropriate collocation methods will be used to solve the singular integral equations for cases with flat and cylindrical punches. Finally, a theoretical analysis will give answers to the above mentioned questions.

\section{Model of a half-space with a graded layer subjected to concentrated forces}

The model shown in Fig. 1a is considered first, in which both a normal concentrated line force $P$ and a tangential concentrated line force $Q$ act on the surface of a graded layer, which is coated on a homogeneous half-space. We assume that Poisson's ratios of both the layer and half-space are equal to a constant $v$. The shear modulus of the layer varies linearly according to the following law:

$$
\mu(y)=\mu_{2}+\mu^{*} y, \quad \mu^{*}=\left(\mu_{1}-\mu_{2}\right) / h, \quad(0 \leq y \leq h),
$$

where $\mu_{2}$ is the shear modulus of the half-space and ensures the continuity at the interface, $y=0 . h$ denotes the thickness of the layer, $\mu^{*}$ is a constant increment of the shear modulus, and $\mu_{1}$ is the shear modulus of the graded layer surface.

Due to the plane strain condition, Hooke's law in the region $0 \leq y \leq h$ can be written as

$$
\begin{aligned}
\varepsilon_{1 x x} & =\frac{1-v}{2 \mu(y)}\left(\sigma_{1 x x}-\frac{v}{1-v} \sigma_{1 y y}\right), \\
\varepsilon_{1 y y} & =\frac{1-v}{2 \mu(y)}\left(\sigma_{1 y y}-\frac{v}{1-v} \sigma_{1 x x}\right), \\
\gamma_{1 x y} & =\frac{1}{\mu(y)} \sigma_{1 x y},
\end{aligned}
$$

where the subscript " 1 " denotes the layer material. 


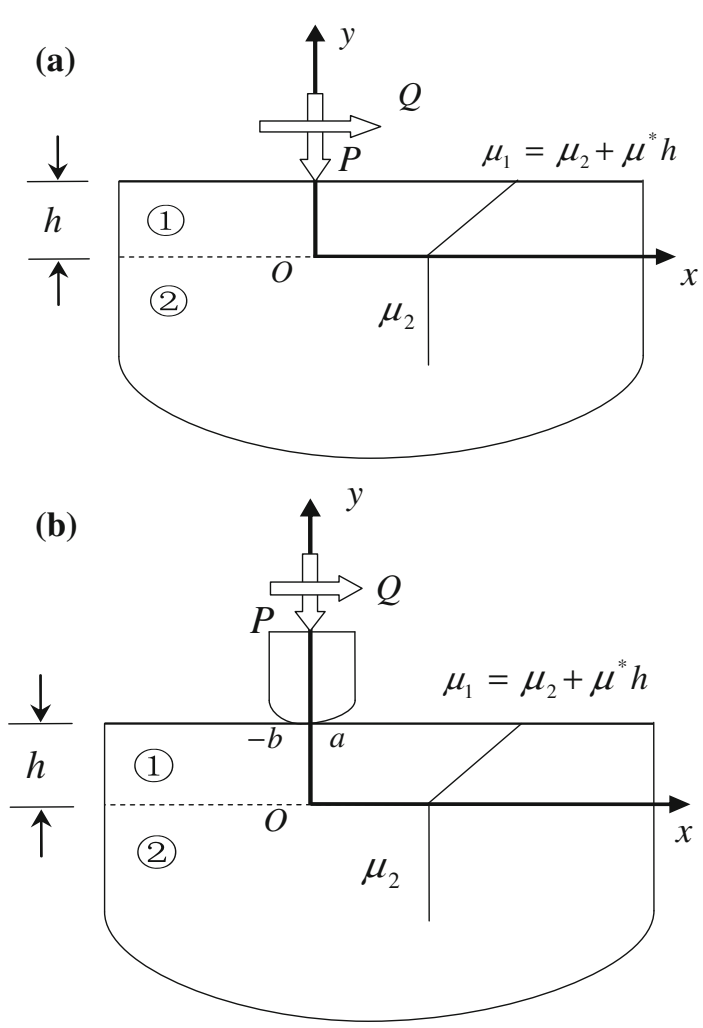

Fig. 1 Schematics of the contact model for a half-space coated with a linear graded layer a loaded by both a concentrated normal load and a tangential one; $\mathbf{b}$ loaded by a rigid punch with an arbitrary shape

The equation of strain compatibility is

$$
\frac{\partial \varepsilon_{1 x x}^{2}}{\partial y^{2}}+\frac{\partial \varepsilon_{1 y y}^{2}}{\partial x^{2}}=\frac{\partial \gamma_{1 x y}^{2}}{\partial x \partial y} .
$$

Introducing an Airy stress function $F_{1}(x, y)$ as follows:

$$
\sigma_{1 x x}=\frac{\partial^{2} F_{1}}{\partial y^{2}}, \quad \sigma_{1 x y}=-\frac{\partial^{2} F_{1}}{\partial x \partial y}, \quad \sigma_{1 y y}=\frac{\partial^{2} F_{1}}{\partial x^{2}}
$$

and substituting Eqs. (2) and (4) into (3) yields

$$
\frac{\partial^{4} F_{1}}{\partial x^{4}}+2 \frac{\partial^{4} F_{1}}{\partial x^{2} \partial y^{2}}+\frac{\partial^{4} F_{1}}{\partial y^{4}}-\frac{2 \mu^{\prime}}{\mu} \frac{\partial^{3} F_{1}}{\partial y^{3}}-\frac{2 \mu^{\prime}}{\mu} \frac{\partial^{3} F_{1}}{\partial x^{2} \partial y}+\frac{2 \mu^{\prime 2}}{\mu^{2}} \frac{\partial^{2} F_{1}}{\partial y^{2}}-\frac{2 \mu^{\prime 2}}{\mu^{2}} \frac{v}{1-v} \frac{\partial^{2} F_{1}}{\partial x^{2}}=0,
$$

where the superscript "“" indicates the differentiation with respect to $y$.

Applying Fourier integral transformation to (5) with respect to $x$ leads to

$$
\begin{gathered}
\frac{\mathrm{d}^{4} \tilde{F}_{1}}{\mathrm{~d} y^{4}}-2 s^{2} \frac{\mathrm{d}^{2} \tilde{F}_{1}}{\mathrm{~d} y^{2}}+s^{4} \tilde{F}-\frac{2 \mu^{*}}{\mu_{0}+\mu^{*} y} \frac{\mathrm{d}^{3} \tilde{F}_{1}}{\partial y^{3}}-\frac{2 \mu^{*} s^{2}}{\mu_{0}+\mu^{*} y} \frac{\mathrm{d} \tilde{F}_{1}}{\mathrm{~d} y} \\
+\frac{2 \mu^{* 2}}{\left(\mu_{0}+\mu^{*} y\right)^{2}} \frac{\mathrm{d}^{2} \tilde{F}_{1}}{\mathrm{~d} y^{2}}-\frac{2 \mu^{* 2}}{\left(\mu_{0}+\mu^{*} y\right)^{2}} \frac{v}{1-v} s^{2} \tilde{F}_{1}=0,
\end{gathered}
$$

where " " indicates the Fourier transformation. Assuming

$$
\xi=2 s\left(\mu_{2}+\mu^{*} y\right) / \mu^{*}, \quad \tilde{F}_{1}=\xi \phi(\xi) / 2,
$$


then, Eq. (6) is reduced to be a Whittaker one,

$$
\frac{\mathrm{d}^{4} \phi}{\mathrm{d} y^{4}}+\frac{2}{\xi} \frac{\mathrm{d}^{3} \phi}{\mathrm{d} y^{3}}-\left(\frac{1}{2}+\frac{4}{\xi^{2}}\right) \frac{\mathrm{d}^{2} \phi}{\mathrm{d} y^{2}}+\left(\frac{4}{\xi^{3}}-\frac{1}{2 \xi}\right) \frac{\mathrm{d} \phi}{\mathrm{d} y}+\left(\frac{1}{16}+\frac{1-\gamma^{2}}{\xi^{2}}\right) \phi=0,
$$

where $\gamma=\sqrt{(1-2 v) /(2-2 v)}$. The solution to Eq. (8) could be found as [31]

$$
\phi=A_{11} W_{\gamma, 1.5}(\xi)+A_{12} W_{-\gamma, 1.5}(\xi)+A_{13} W_{\gamma, 1.5}(-\xi)+A_{14} W_{-\gamma, 1.5}(-\xi),
$$

where $A_{1 i}(i=1,2,3,4)$ are unknown coefficients and $W_{ \pm \gamma, 1.5}( \pm \xi)$ are Whittaker functions. Substituting Eq. (9) into Eq. (7) yields

$$
\begin{aligned}
\tilde{F}_{1} & =\left(A_{11} W_{\gamma, 1.5}(\xi)+A_{12} W_{-\gamma, 1.5}(\xi)+A_{13} W_{\gamma, 1.5}(-\xi)+A_{14} W_{-\gamma, 1.5}(-\xi)\right) \xi / 2 \\
& =A_{11} \tilde{\phi}_{1}(\xi)+A_{12} \tilde{\phi}_{2}(\xi)+A_{13} \tilde{\phi}_{3}(\xi)+A_{14} \tilde{\phi}_{4}(\xi) .
\end{aligned}
$$

The Fourier transformed displacements and stress components can be written as a matrix form as

$$
\left\{S_{1}\right\}=\left[T_{1}(y)\right]\left\{A_{1}\right\}=\left[T_{11}(y) T_{12}(y) T_{13}(y) T_{14}(y)\right]\left\{A_{1}\right\},
$$

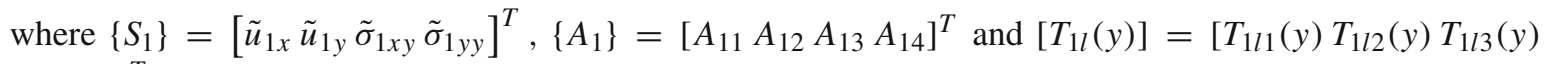
$\left.T_{1 l 4}(y)\right]^{T}$ with

$$
\begin{aligned}
& T_{1 l 1}(y)=-\frac{\mathrm{i}(1-v)}{2 \mu(y) s} \frac{\mathrm{d}^{2} \tilde{\phi}_{1 l}}{\mathrm{~d} y^{2}}-\frac{\mathrm{i} v \mathrm{~s}}{2 \mu(y)} \tilde{\phi}_{1 l}, \\
& T_{1 l 2}(y)=\frac{1-v}{2 \mu(y) s^{2}} \frac{\mathrm{d}^{3} \tilde{\phi}_{1 l}}{\mathrm{~d} y^{3}}-\frac{\mu^{*}(1-v)}{2 \mu^{2}(y) s^{2}} \frac{\mathrm{d}^{2} \tilde{\phi}_{1 l}}{\mathrm{~d} y^{2}}-\frac{2-v}{2 \mu(y)} \frac{\mathrm{d} \tilde{\phi}_{1 l}}{\mathrm{~d} y}-\frac{\mu^{*} v}{2 \mu^{2}(y)} \tilde{\phi}_{1 l}, \\
& T_{1 l 3}(y)=-\mathrm{i} s \frac{\mathrm{d} \tilde{\phi}_{1 l}}{\mathrm{~d} y}, \\
& T_{1 / 4}(y)=-s^{2} \tilde{\phi}_{1 l}, \quad l=1,2,3,4 .
\end{aligned}
$$

The superscript " $T$ " denotes transposition of a matrix.

For the lower homogenous half-space, which is denoted as material "2", the Airy stress function $F_{2}$ should satisfy a bi-harmonic equation as

$$
\nabla^{4} F_{2}=0
$$

Similar to the above, we have

$$
\left\{S_{2}\right\}=\left[T_{2}(y)\right]\left\{A_{2}\right\}
$$

where $\left\{A_{2}\right\}=\left[A_{21}, A_{22}\right]^{T}$ and

$$
\left[T_{2}(y)\right]=\left[\begin{array}{llll}
s / 2 \mathrm{i} \mu_{2} & -|s| / 2 \mu_{2} & -\mathrm{i} s & -s^{2} \\
{[y s+2(1-v)|s| / s] / 2 \mathrm{i} \mu_{2}} & (1-2 v-y|s|) / 2 \mu_{2} & -\mathrm{i} s(y|s|+1) & -s^{2} y
\end{array}\right]^{T} \mathrm{e}^{|s| y} .
$$

The boundary condition at the interface $y=0$ can be written as

$$
\left\{S_{1}\right\}-\left\{S_{2}\right\}=\{0\},
$$

and on the coating surface $y=h$, we have

$$
\left\{\begin{array}{l}
\sigma_{1 x y}(x, h)=-\delta(x) Q \\
\sigma_{1 y y}(x, h)=-\delta(x) P
\end{array}\right.
$$

where $\delta(x)$ is the delta function.

Equation (16) can also be expressed in a matrix form as

$$
\left[B_{1}\right]\left[T_{1}(h)\right]\left\{A_{1}\right\}=\{-Q,-P\}^{T}
$$


where

$$
\left[B_{1}\right]=\left[\begin{array}{llll}
0 & 0 & 1 & 0 \\
0 & 0 & 0 & 1
\end{array}\right]
$$

Equations (15) and (17) constitute a recurrence relation which, upon substitution in (11) and (13), may yield the expression of $\left\{A_{1}\right\}$ in terms of $\{Q, P\}^{T}$,

$$
\left\{\begin{array}{l}
\left\{A_{1}\right\}=-\left[V_{1}\right][K]^{-1}\{Q, P\}^{T} \\
{\left[V_{1}\right]=\left[T_{1}(0)\right]^{-1}\left[T_{2}(0)\right],[K]=\left[B_{1}\right]\left[T_{1}(h)\right]\left[V_{1}\right] .}
\end{array}\right.
$$

Substituting Eq. (18) into (11) and taking the inverse Fourier transformation leads to

$$
\left[u_{x 1}, u_{y 1}, \sigma_{x y 1}, \sigma_{y y 1}\right]^{T}=\frac{1}{2 \pi} \int_{-\infty}^{\infty}[M]\{Q, P\}^{T} \mathrm{e}^{\mathrm{i} s x} \mathrm{~d} s
$$

where

$$
[M]=-\left[T_{1}(y)\right]\left[V_{1}\right][K]^{-1}
$$

is a transfer matrix for the layer. The displacements at $y=h$ can be written as

$$
\left\{\begin{array}{l}
u_{x h} \\
u_{y h}
\end{array}\right\}=\frac{1}{2 \pi} \int_{-\infty}^{\infty} m(s, h)\{Q, P\}^{T} \mathrm{e}^{\mathrm{i} s x} \mathrm{~d} s
$$

where

$$
m(s, h)=\left[B_{2}\right][M(s, h)],\left[B_{2}\right]=\left[\begin{array}{llll}
1 & 0 & 0 & 0 \\
0 & 1 & 0 & 0
\end{array}\right] .
$$

Considering the asymptotic behavior of Whittaker functions for large arguments [31], one can readily prove that

$$
\lim _{s \rightarrow+\infty} \operatorname{sm}(s, h)=\left[\begin{array}{cc}
\alpha_{1} & -\mathrm{i} \alpha_{2} \\
\mathrm{i} \alpha_{2} & \alpha_{1}
\end{array}\right]
$$

where

$$
\alpha_{1}=\frac{v-1}{\mu_{1}}, \quad \alpha_{2}=\frac{2 v-1}{2 \mu_{1}} .
$$

Denoting

$$
\Lambda=\frac{1}{s}\left[\begin{array}{ll}
\operatorname{sign}(s) \alpha_{1} & -\mathrm{i} \alpha_{2} \\
\mathrm{i} \alpha_{2} & \operatorname{sign}(s) \alpha_{1}
\end{array}\right]
$$

Eq. (20) can be rewritten as

$$
\left\{\begin{array}{l}
u_{x h} \\
u_{y h}
\end{array}\right\}=\frac{1}{2 \pi} \int_{-\infty}^{\infty} \Lambda\{Q, P\}^{T} \mathrm{e}^{\mathrm{i} s x} \mathrm{~d} s+\frac{1}{2 \pi} \int_{-\infty}^{\infty}[m(s, h)-\Lambda]\{Q, P\}^{T} \mathrm{e}^{\mathrm{i} s x} \mathrm{~d} s .
$$

Considering the following properties of the elements in matrix $m(s)$ :

$$
m_{i j}(-s)=(-1)^{i+j} m_{i j}(s), \quad i, j=1,2,
$$

and using the relations

$$
\int_{0}^{\infty} \frac{\cos (s x)}{s} \mathrm{~d} s=\ln |x|, \quad \int_{0}^{\infty} \frac{\sin (s x)}{s} \mathrm{~d} s=\frac{\pi}{2} \operatorname{sign}(x)
$$


yields

$$
\left\{\begin{aligned}
u_{x h}(x)= & \frac{\alpha_{2} P}{2} \operatorname{sign}(x)-\frac{\alpha_{1} Q}{\pi} \ln |x|+\frac{P}{\pi} \int_{0}^{\infty}\left[\operatorname{im} m_{12}(s)-\alpha_{2} / s\right] \sin (s x) \mathrm{d} s \\
& +\frac{Q}{\pi} \int_{0}^{\infty}\left[m_{11}(s)-\alpha_{1} / s\right] \cos (s x) \mathrm{d} s, \\
u_{y h}(x)= & -\frac{\alpha_{1} P}{\pi} \ln |x|-\frac{\alpha_{2} Q}{2} \operatorname{sign}(x)+\frac{P}{\pi} \int_{0}^{\infty}\left[m_{22}(s)-\alpha_{1} / s\right] \cos (s x) \mathrm{d} s \\
& +\frac{Q}{\pi} \int_{0}^{\infty}\left[\operatorname{im} m_{21}(s)+\alpha_{2} / s\right] \sin (s x) \mathrm{d} s .
\end{aligned}\right.
$$

The above equations denote the relation of the surface displacements $u_{x h}, u_{y h}$ and the linear concentrated forces $P, Q$ in the model as shown in Fig. 1a.

\section{Stamp model of a rigid punch with an arbitrary profile}

Using the above relation in Eq. (25), we will investigate a stamp model as shown in Fig. 1b, where a rigid punch contacts an elastic half-space with a graded surface layer.

The problem will become a typically mixed-boundary-value one, in which the displacement components can be given by the punch profile within the contact region. Supposing the normal and tangential pressures at the contact interface are $p(x)$ and $q(x)$, respectively, the displacements at the contact interface can be found by the superposition theorem as

$$
\begin{aligned}
u_{x h}(x)= & -\frac{\alpha_{1}}{\pi} \int_{-b}^{a} \ln |x-t| q(t) \mathrm{d} t+\frac{1}{\pi} \int_{-b}^{a} q(t) I_{1}(x, t) \mathrm{d} t \\
& +\frac{\alpha_{2}}{2}\left[\int_{-b}^{x} p(t) \mathrm{d} t-\int_{x}^{a} p(t) \mathrm{d} t\right]+\frac{1}{\pi} \int_{-b}^{a} p(t) I_{2}(x, t) \mathrm{d} t, \\
u_{y h}= & -\frac{\alpha_{2}}{2}\left[\int_{-b}^{x} q(t) \mathrm{d} t-\int_{x}^{a} q(t) \mathrm{d} t\right]+\frac{1}{\pi} \int_{-b}^{a} q(t) I_{3}(x, t) \mathrm{d} t \\
& -\frac{\alpha_{1}}{\pi} \int_{-b}^{a} \ln |x-t| p(t) \mathrm{d} t+\frac{1}{\pi} \int_{-b}^{a} p(t) I_{4}(x, t) \mathrm{d} t
\end{aligned}
$$

where

$$
\begin{aligned}
& I_{1}(x, t)=\int_{0}^{\infty}\left[m_{11}(s)-\frac{\alpha_{1}}{s}\right] \cos [s(x-t)] \mathrm{d} s, \\
& I_{2}(x, t)=\mathrm{i} \int_{0}^{\infty}\left[m_{12}(s)+\mathrm{i} \frac{\alpha_{2}}{s}\right] \sin [s(x-t)] \mathrm{d} s, \\
& I_{3}(x, t)=\mathrm{i} \int_{0}^{\infty}\left[m_{21}(s)-\mathrm{i} \frac{\alpha_{2}}{s}\right] \sin [s(x-t)] \mathrm{d} s, \\
& I_{4}(x, t)=\int_{0}^{\infty}\left[m_{22(s)-\frac{\alpha_{1}}{s}}\right] \cos [s(x-t)] \mathrm{d} s .
\end{aligned}
$$


The partial derivative of Eq. (26) with respect to $x$ yields

$$
\begin{gathered}
\alpha_{2} p(x)+\frac{1}{\pi} \int_{-b}^{a}\left[Q_{1}(x, t)+\frac{\alpha_{1}}{t-x}\right] q(t) \mathrm{d} t+\frac{1}{\pi} \int_{-b}^{a} p(t) Q_{2}(x, t) \mathrm{d} t=g_{1}(x), \\
-\alpha_{2} q(x)+\frac{1}{\pi} \int_{-b}^{a}\left[Q_{4}(x, t)+\frac{\alpha_{1}}{t-x}\right] p(t) \mathrm{d} t+\frac{1}{\pi} \int_{-b}^{a} q(t) Q_{3}(x, t) \mathrm{d} t=g_{2}(x)
\end{gathered}
$$

where $g_{1}(x)=\partial u_{x h}(x) / \partial x, g_{2}(x)=\partial u_{y h}(x) / \partial x$ and $Q_{j}(x, t)=\partial I_{j}(x, t) / \partial x,(j=1,2,3,4) . b$ and $a$ are the left and right contact lengths, respectively. Equations (27) and (28) are coupled integral ones with Cauchy singularity, in which the unknown contact pressures $p(x)$ and $q(x)$ need to be determined whereas $u_{y h}(x),(-b<x<a)$ can be deduced from the punch profile.

In this study, we assume that the friction between the punch and the substrate belongs to the Coulomb type with a constant friction coefficient $\eta$, i.e.,

$$
\begin{aligned}
& \sigma_{y y}(x, h)=-p(x), \\
& \sigma_{x y}(x, h)=-\eta p(x), \quad(-b<x<a),
\end{aligned}
$$

then, $p(x)$, as an unknown function, can be obtained from the following equation:

$$
-\alpha_{2} \eta p(x)+\frac{1}{\pi} \int_{-b}^{a}\left[Q_{4}(x, t)+\eta Q_{3}(x, t)+\frac{\alpha_{1}}{t-x}\right] p(t) \mathrm{d} t=g_{2}(x),
$$

with the following requirement:

$$
\int_{-b}^{a} p(t) \mathrm{d} t=P
$$

By introducing the following normalized quantities:

$$
t=\frac{a+b}{2} \theta+\frac{a-b}{2}, \quad x=\frac{a+b}{2} \varsigma+\frac{a-b}{2}, \quad-b<(t, x)<a, \quad-1<(\theta, \varsigma)<1,
$$

Eqs. (30) and (31) then can be expressed as

$$
\begin{gathered}
-\alpha_{2} \eta p(\varsigma)+\frac{\alpha_{1}}{\pi} \int_{-1}^{1} \frac{p(\theta)}{\theta-\varsigma} \mathrm{d} \theta+\frac{a+b}{2 \pi} \int_{-1}^{1}\left[Q_{4}(\varsigma, \theta)+\eta Q_{3}(\varsigma, \theta)\right] p(\theta) \mathrm{d} t=g_{2}(\varsigma), \\
\int_{-1}^{1} p(\theta) \mathrm{d} \theta=2 P /(a+b) .
\end{gathered}
$$

The numerical method proposed by Krenk [32] can be used to solve the above integral equations (33) and (34). Details are given as follows.

Assume

$$
p(\varsigma)=f(\varsigma) w(\varsigma), \quad-1<\varsigma<1,
$$

where $w(\varsigma)$ is the weight function of $p(\varsigma)$ and may be determined as

$$
\begin{aligned}
w(\varsigma) & =(1-\varsigma)^{\beta_{1}}(1+\varsigma)^{\beta_{2}}, \\
\beta_{1} & =\frac{1}{\pi} \arctan \left(\frac{\alpha_{1}}{\alpha_{2} \eta}\right)+N_{0}, \quad \beta_{2}=-\frac{1}{\pi} \arctan \left(\frac{\alpha_{1}}{\alpha_{2} \eta}\right)+M_{0},
\end{aligned}
$$


where $N_{0}$ and $M_{0}$ are arbitrary integers (positive, zero, or negative) and are determined from the physics of the problem. The index of the integral equations is defined as

$$
\kappa=-\left(\beta_{1}+\beta_{2}\right)=-\left(N_{0}+M_{0}\right) .
$$

In order to get integrable singularities, $\kappa$ should be restricted to $-1,0$, or 1 [1]. Equations (33) and (34) can be reduced to

$$
\begin{gathered}
\sum_{l=1}^{M} W_{l}^{M} f\left(\theta_{l}\right)\left[\frac{\alpha_{1}}{\theta_{l}-\varsigma_{r}}+\frac{a+b}{2}\left[Q_{4}\left(\varsigma_{r}, \theta_{l}\right)+\eta Q_{3}\left(\varsigma_{r}, \theta_{l}\right)\right]\right]=g_{2}\left(\varsigma_{r}\right), \\
\sum_{l=1}^{M} W_{l}^{M} f\left(\theta_{l}\right)=\frac{2 P}{\pi(a+b)},
\end{gathered}
$$

where $M$ is the total number of the discrete points of $f\left(\theta_{l}\right)$ in $(-1,1) ; \theta_{l}, \varsigma_{r}$, and $W_{l}^{M}$ are, respectively, determined by

$$
\begin{aligned}
& P_{M}^{\left(\beta_{1}, \beta_{2}\right)}\left(\theta_{l}\right)=0, \quad l=1,2, \ldots, M, \\
& P_{M-\kappa}^{\left(-\beta_{1},-\beta_{2}\right)}\left(\varsigma_{r}\right)=0, \quad r=1,2, \ldots, M-\kappa, \\
& W_{l}^{M}=-2^{\kappa} \frac{\Gamma\left(\beta_{1}\right) \Gamma\left(1-\beta_{1}\right)}{\pi} \frac{P_{M-\kappa}^{\left(-\beta_{1},-\beta_{2}\right)}\left(\theta_{l}\right)}{P_{M}^{\left(\beta_{1}, \beta_{2}\right)^{\prime}}\left(\theta_{l}\right)},
\end{aligned}
$$

where $\Gamma()$ is the Gamma function and $P_{M}^{\left(\beta_{1}, \beta_{2}\right)}()$ is the Jacobi polynomial of degree $M$.

\section{Examples}

\subsection{Rigid flat punch case}

Consider the contact problem as shown in Fig. 1b, if the punch is a flat one, i.e.,

$$
\frac{\partial u_{y h}}{\partial x}=0 .
$$

Then, we have

$$
g(\varsigma)=0, \quad b=a .
$$

The physics of the problem requires that both $\beta_{1}$ and $\beta_{2}$ should be negative. Considering Eq. (38), we should select $N_{0}=-1$ and $M_{0}=0$ [9]. Then, numerical results can be obtained by solving Eqs. (39) and (40).

After $f\left(\theta_{l}\right)$ is determined, the contact stresses can be found as

$$
\begin{aligned}
\frac{\sigma_{y y}(x, h)}{\sigma_{0}} & =-\frac{p(x)}{\sigma_{0}}, \\
\sigma_{0} & =\frac{P}{2 a}
\end{aligned}
$$

where

$$
p(x)=f(x / a)(1-x / a)^{\beta_{1}}(1+x / a)^{\beta_{2}} .
$$

Solving the problem yields the stress intensity factors at points $x= \pm a$,

$$
\begin{aligned}
K_{1}(a) & =\lim _{x \rightarrow a} \frac{p(x)}{2^{\beta_{2}}(a-x)^{\beta_{1}}}=f(1) a^{1+\beta_{2}}, \\
K_{1}(-a) & =\lim _{x \rightarrow a} \frac{p(x)}{2^{\beta_{1}}(a+x)^{\beta_{2}}}=f(-1) a^{1+\beta_{1}} .
\end{aligned}
$$


4.2 Rigid cylindrical punch case

Consider the plane strain problem with a cylindrical punch, where the punch profile is given by

$$
u_{y h}=\left(R-\sqrt{R^{2}-x^{2}}\right)-\delta_{0}, \quad \frac{\partial u_{y h}}{\partial x}=\frac{x}{\sqrt{R^{2}-x^{2}}}, \quad-b<x<a,
$$

where $R$ is the radius of the punch; $\delta_{0}$ is the maximum indentation depth appearing at $x=0$. Without considering the interfacial adhesion, the pressure at the contact edges should vanish, i.e.,

$$
p(-b)=p(a)=0 .
$$

Because of smooth contacts at both ends $x=-b$ and $x=a$, the physics of the problem requires that both $\beta_{1}$ and $\beta_{2}$ be positive. This, by considering Eq. (38), may be fulfilled by selecting $N_{0}=0$ and $M_{0}=1$ [26].

It is noticed that for a cylindrical punch problem the solution of Eqs. (39) and (40) must satisfy a consistency condition [33],

$$
\int_{-1}^{1} \frac{S(\varsigma) \mathrm{d} \varsigma}{(1-\varsigma)^{\beta_{1}}(1+\varsigma)^{\beta_{2}}}=0
$$

where

$$
S(\varsigma)=-\alpha_{2} \eta p(\varsigma)+\frac{\alpha_{1}}{\pi} \int_{-1}^{1} \frac{p(\theta)}{\theta-\varsigma} \mathrm{d} \theta .
$$

According to Krenk [32], it is easily proved that Eq. (49) is spontaneously satisfied.

\section{Special cases}

If the coating thickness tends to be infinity, then $\mu^{*}=0$. The present problem becomes approximately a special case, i.e., a rigid punch in contact with an elastic homogeneous half-space with a constant shear modulus $\mu$ and Poisson's ratio $v[9,26]$. Closed-form solutions for the special case can be easily found from the above layer problem, which is a relatively general model.

For the special case, Eq. (30) can be reduced to

$$
-\alpha_{2} \eta p(x)+\frac{1}{\pi} \int_{-b}^{a} \frac{\alpha_{1}}{t-x} p(t) \mathrm{d} t=g_{2}(x)
$$

where

$$
g_{2}(x)=\partial u_{y h}(x) / \partial x .
$$

For the special case with a rigid flat punch, we have

$$
g_{2}(x)=0, \quad b=a .
$$

Combining Eqs. (51) and (53) leads to the closed-form solution

$$
p(x)=-\frac{2 \sigma_{0} \sin \pi \beta_{1}}{\pi}\left(1-\frac{x}{a}\right)^{\beta_{1}}\left(1+\frac{x}{a}\right)^{\beta_{2}}, \quad-a<x<a .
$$

Then, the stress intensity factors at the contact edges can be found,

$$
\begin{aligned}
K_{1}(a) & =-\frac{2 \sigma_{0}}{\pi a^{\beta_{1}}} \sin \pi \beta_{1}, \\
K_{1}(-a) & =-\frac{2 \sigma_{0}}{\pi a^{\beta_{2}}} \sin \pi \beta_{1} .
\end{aligned}
$$


For the special case with a plane strain rigid cylindrical punch, the punch profile is usually approximated to be a parabolic function. Then, we have,

$$
u_{y h}=-\delta_{0}+\frac{x^{2}}{2 R}, \quad \frac{\partial u_{y h}}{\partial x}=\frac{x}{R}, \quad-b<x<a .
$$

Substituting Eq. (57) into Eq. (51) yields a closed-form solution for the normal traction in the contact region,

$$
p(x)=\frac{\mu_{1}}{1-v} \frac{\sin \pi \beta_{1}}{R}(a-x)^{\beta_{1}}(x+b)^{\beta_{2}}, \quad-b<x<a .
$$

Both the closed-form solutions are identical to those in $[9,26]$.

\section{Results and discussions}

In what follows, numerical results for the present general case are obtained. A comprehensive discussion is conducted to reveal the influences of parameters, such as the variation law of modulus, thickness of graded layer, ratio of stiffness, frictional coefficient, etc., on the stress intensity factors and stress distributions for both flat and cylindrical stamp cases.

\subsection{Stress singularity and stress intensity factor}

Table 1 shows some results of the stress singularities $\beta_{1}$ and $\beta_{2}$ at the leading $(x=a)$ and the trailing $(x=-a)$ edges, respectively, and stress intensify factors obtained for a flat punch by assuming $a / h=0.5, v=0.3$ with different ratios of stiffness $\mu_{2} / \mu_{1}$ and different values of frictional coefficient $\eta$. For comparison, not only the case of a graded layer with a linear variation law of the shear modulus $\mu(y)=\mu_{2}+\mu^{*} y,\left(\mu^{*}=\left(\mu_{1}-\mu_{2}\right) / h\right)$, but also that with an exponential one $\mu(y)=\mu_{2} \mathrm{e}^{\delta y},\left(\delta=\log \left(\mu_{1} / \mu_{2}\right) / h\right)$ proposed by Guler and Erdogan $[9,26]$ is included. One should note that in contact problems $\beta_{1}$ and $\beta_{2}$ are independent of the material inhomogeneity parameters (i.e., $\mu^{*}$ and $\delta$ ) and depend only on the coefficient of friction $\eta$ and the value of Poisson's ratio $v$ on the surface $y=h$. For a flat stamp case, it is found that for a fixed value of $v$, as $\eta$ approaches zero, $\beta_{1} \rightarrow-1 / 2, \beta_{2} \rightarrow-1 / 2$, which corresponds to the known singularity for a frictionless stamp case. As the frictional coefficient increases, the singularity at the trailing edge of the flat punch is stronger than that at the leading edge, which means larger stress concentration near the trailing edge. If $\eta$ takes a relatively large value, we find that $\beta_{1} \rightarrow 0$ and $\beta_{2} \rightarrow-1$.

In addition, from Table 1 , one can see that for stiffer substrate cases, i.e., $\mu_{2}>\mu_{1}$, the stress intensity factors are smaller than in cases with a softer substrate, i.e., $\mu_{2}<\mu_{1}$. The Table also shows that different variation laws of elastic modulus should have considerable influence on the contact behavior. The stress intensity factors in cases with graded layers of a linear variation law are obviously smaller than that of an exponential variation one.

Tables 2a, $\mathrm{b}$ show the variation of non-dimensional stress intensity factors $\frac{k_{1}(a)}{P a^{\beta_{2}}}$ for cases with frictionless coefficient $\eta=0$, fixed stiffness ratios $\mu_{2} / \mu_{1}=8$ and $\mu_{2} / \mu_{1}=1 / 8$, and different non-dimensional contact radius $a / h$. For cases with stiffer substrates, i.e., $\mu_{2}>\mu_{1}$, the stress intensity factors decrease with an increase of $a / h$. However, for cases with softer substrates, the stress intensity factors increase rapidly when the linear graded layer becomes thinner.

Table 1 Stress singularities $\beta_{1}$ and $\beta_{2}$ near the flat punch edges and the stress intensity factors in models with a linear graded

\begin{tabular}{|c|c|c|c|c|c|c|c|}
\hline \multirow[t]{2}{*}{$\mu_{2} / \mu_{1}$} & \multirow{2}{*}{$\begin{array}{l}\eta=0.0 \\
\beta_{1}=-0.5 \\
\beta_{2}=-0.5 \\
\frac{K_{1}(a)}{P a^{\beta_{2}}}\end{array}$} & \multicolumn{2}{|c|}{$\begin{array}{l}\eta=0.1 \\
\beta_{1}=-0.4909 \\
\beta_{2}=-0.5091\end{array}$} & \multicolumn{2}{|c|}{$\begin{array}{l}\eta=0.3 \\
\beta_{1}=-0.4728 \\
\beta_{2}=-0.5272\end{array}$} & \multicolumn{2}{|c|}{$\begin{array}{l}\eta=0.5 \\
\beta_{1}=-0.4548 \\
\beta_{2}=-0.5452\end{array}$} \\
\hline & & $\frac{K_{1}(a)}{P a^{\beta_{2}}}$ & $\frac{K_{1}(-a)}{P a^{\beta_{1}}}$ & $\frac{K_{1}(a)}{P a^{\beta_{2}}}$ & $\frac{K_{1}(-a)}{P a^{\beta_{1}}}$ & $\frac{K_{1}(a)}{P a^{\beta_{2}}}$ & $\frac{K_{1}(-a)}{P a^{\beta_{1}}}$ \\
\hline 8 (Exp) & 0.2086 & 0.1973 & 0.2199 & 0.1754 & 0.2422 & 0.1549 & 0.2635 \\
\hline 8 (Linear) & 0.1779 & 0.1714 & 0.1850 & 0.1572 & 0.2022 & 0.1451 & 0.2181 \\
\hline 1 & 0.3183 & 0.3182 & 0.3182 & 0.3171 & 0.3171 & 0.3151 & 0.3151 \\
\hline 1/8 (Exp) & 0.6011 & 0.6178 & 0.5844 & 0.6510 & 0.5511 & 0.6834 & 0.5185 \\
\hline 1/8 (Linear) & 0.5427 & 0.5474 & 0.5377 & 0.5555 & 0.5266 & 0.5619 & 0.5145 \\
\hline
\end{tabular}
layer and an exponential one for $a / h=0.5, v=0.3$, different frictional coefficients $\eta$ and different stiffness ratios $\mu_{2} / \mu_{1}$ 
Table 2 Stress intensity factors for a frictionless flat punch case (a) with a linear graded layer and $\mu_{2} / \mu_{1}=8, v=0.3$; (b) with a linear graded layer and $\mu_{2} / \mu_{1}=1 / 8, v=0.3$

\begin{tabular}{lllll}
\hline$a / h$ & 0.005 & 0.01 & 0.1 & 0.5 \\
\hline $\mathrm{a}$ & 0.3162 & 0.3111 & 0.2471 & 0.1779 \\
$\mathrm{~b}$ & $\frac{K_{1}(a)}{P a^{\beta_{2}}}$ & 0.3199 & 0.3515 & 0.5427 \\
$\mathrm{~b}$ & $\frac{K_{1}(a)}{P a^{\beta 2}}$ & 0.3187 & 0.375 & \\
\hline
\end{tabular}

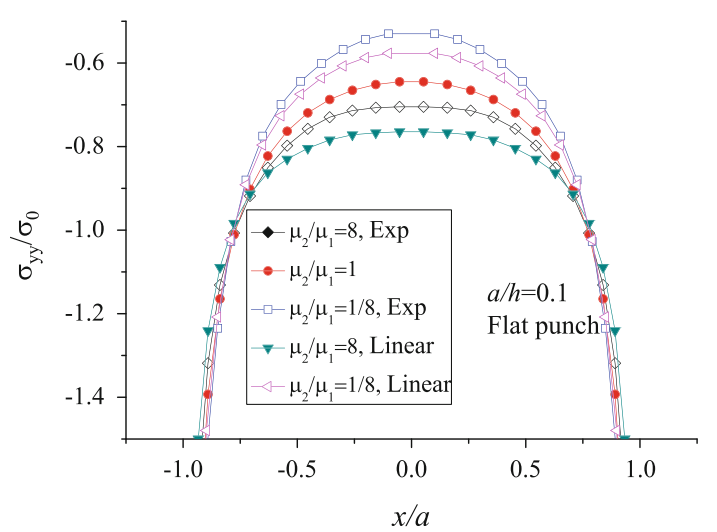

Fig. 2 Distributions of contact pressure in the contact region for frictionless contact models of a rigid flat punch and a linear graded-layered half-space or an exponential graded-layered one, with different values of $\mu_{2} / \mu_{1}$ and fixed $a / h . \sigma_{0}$ is an average compressive stress and is defined as $\sigma_{0}=\frac{P}{2 a}$

\subsection{Interfacial stress distribution}

The distributions of interfacial normal traction are of practical interest, which are analyzed in Figs. 2, 3, 4, and 5 for frictionless models and Fig. 6 corresponding to a frictional one.

Figure 2 shows the distributions of the non-dimensional contact pressure for the frictionless model with a rigid flat punch, in which both the linear variation case and the exponential variation one are investigated with a fixed value of $a / h$ and different ratios of $\mu_{2} / \mu_{1} . \sigma_{0}$ is an average compressive stress, which is defined as $\sigma_{0}=\frac{P}{2 a}$. Figure 2 shows that the contact pressure, as studied in the above, is singular at both edges $x= \pm a$. Due to the frictionless condition, the stress distribution is symmetric with respect to $y=0$. With the increase of $\mu_{2} / \mu_{1}$, the contact pressure in the flat stamp case decreases and becomes more even in the contact area.

Comparing the cases with a linear variation law and an exponential one, one can see that the contact pressure is lower and more uniform in the case with a linearly graded layer than that with an exponentially graded one. These results qualitatively agree with [30], in which a two-dimensional model of a rigid punch contacting an elastic graded half-plane with Young's modulus varying according to a power law was considered. It was found that when the power law is reduced to a linear one, the contact pressure distribution becomes more uniform in the contact region. In addition, comparing to $[10,11]$, both the values of $\frac{K_{1}(a)}{P a^{\beta_{2}}}$ (corresponding to $\eta=0$ ) and the distributions of contact pressure in the present case of $\mu_{2} / \mu_{1}>1$ show a significant improvement of crack suppression characteristics for a linearly graded coating case in contrast to a homogeneous one, i.e., $\mu_{2} / \mu_{1}=1$. The results are also consistent well with the frictionless experimental and FEM results in [10,11].

The effect of graded layer thickness on the contact pressure in the model of a rigid flat punch is shown in Fig. 3a, $b$ for a linear graded layer case. Figure $3 \mathrm{a}$ gives the relation of the contact pressure and the contact width for a stiffer substrate case, i.e., the value of $\mu_{2} / \mu_{1}$ larger than 1 , and different non-dimensional layer thicknesses $h / a$. From Fig. 3a, we find that the contact pressure distributes more uniformly when the graded layer thickness decreases. However, for a softer substrate case, the contrary phenomenon can be found, i.e., the contact pressure distributes more uniformly for an increasing layer thickness as shown in Fig. 3b.

In contrast to Figs. 2 and 3, Figs. 4 and 5 show the distributions of the contact pressure for the frictionless model with a rigid cylindrical punch, and both the linear variation case and the exponential variation one are investigated. From Fig. 4, we find that, with the decrease of $\mu_{2} / \mu_{1}$, the contact pressure in the cylindrical 

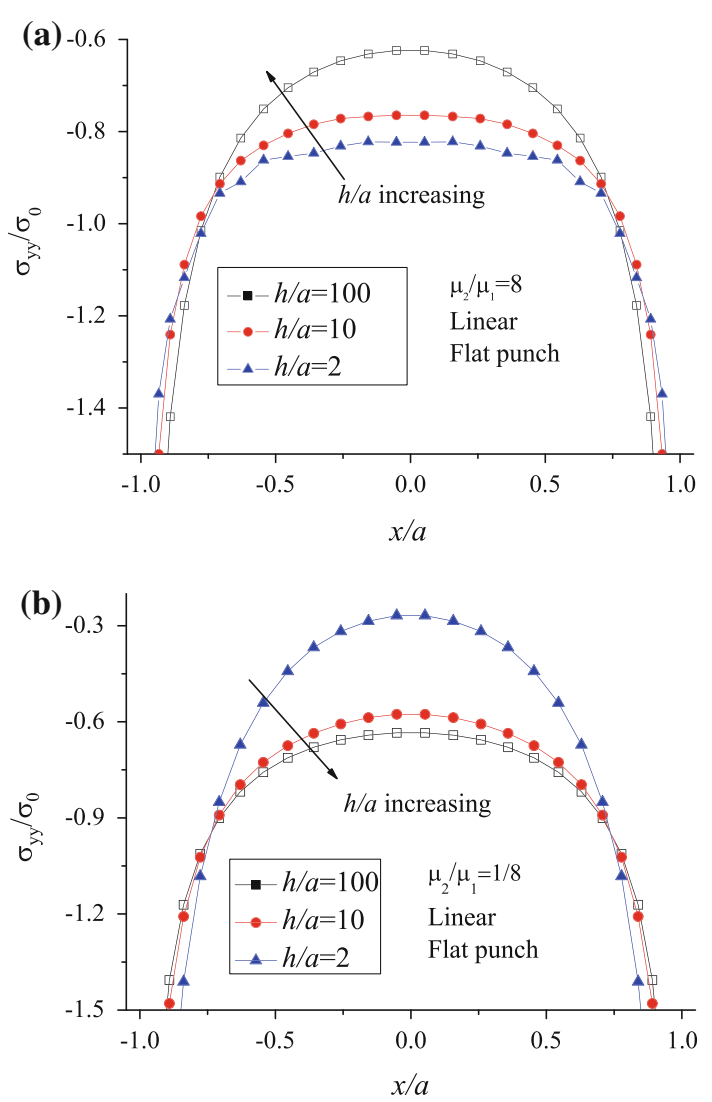

Fig. 3 The effects of $h / a$ on the distributions of contact pressure in the contact region for frictionless contact models of a rigid flat punch and a linear graded-layered half-space with fixed $\mu_{2} / \mu_{1}$. a For $\mu_{2} / \mu_{1}=8$; $\mathbf{b}$ for $\mu_{2} / \mu_{1}=1 / 8$

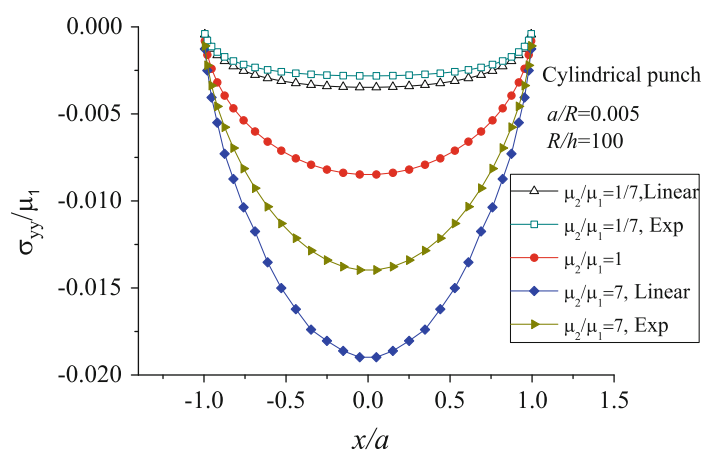

Fig. 4 Distributions of contact pressure in the contact region for frictionless contact models of a rigid cylindrical punch and a linear graded-layered half-space or an exponential graded-layered one, with different values of $\mu_{2} / \mu_{1}$ and fixed $a / R, R / h$

stamp case decreases and becomes more even in the contact area. Comparing the cases with a linear variation law and an exponential one, one can see that the contact pressure is lower and more uniform in the case with an exponentially graded layer than in that with a linearly graded one. All the phenomena in the cylindrical punch case are opposite to those in the flat punch one, which exhibits the effect of the punch profile on the contact behavior.

The effect of the graded layer thickness on the contact pressure in the model of a rigid cylindrical punch is shown in Fig. 5a, b for a linear graded layer case. Figure 5a gives the relation of the normalized contact pressure and the contact width for a stiffer substrate case, i.e., the value of $\mu_{2} / \mu_{1}$ larger than 1 , and different non-dimensional layer thicknesses $h / a$. From Fig. 5a, we find that the contact pressure distributes more uniformly when the graded layer thickness increases with a fixed contact width. However, for a softer substrate 

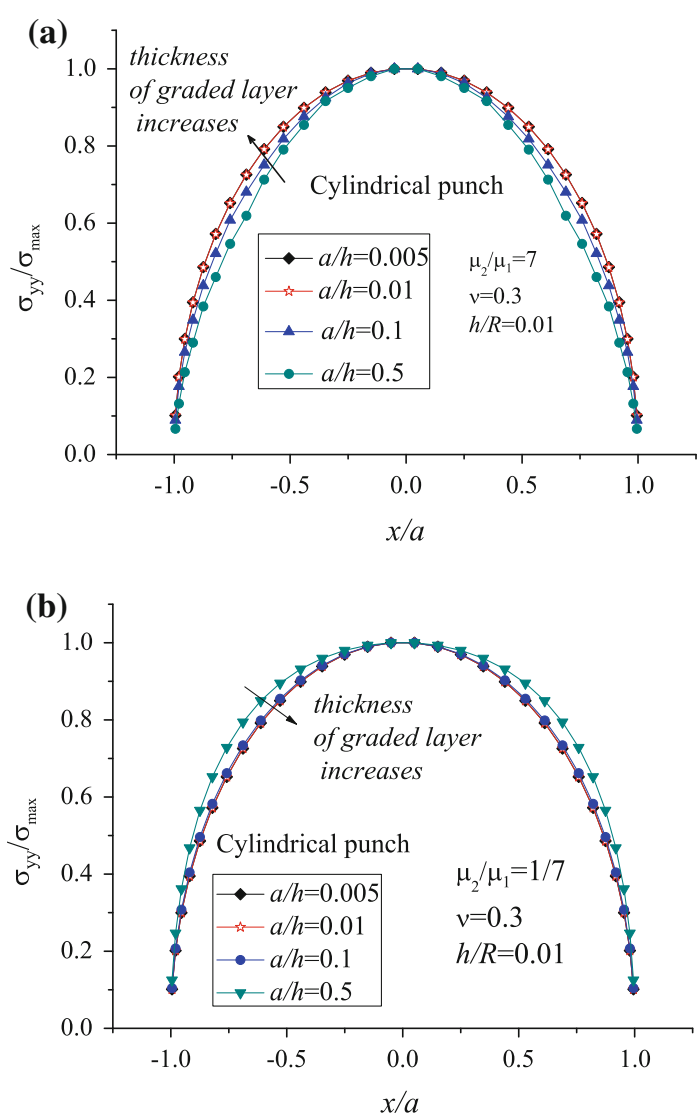

Fig. 5 The effects of $a / h$ on the distributions of the normalized contact pressure in the contact region area for frictionless contact models of a rigid cylindrical punch and a linear graded-layered half-space with fixed $\mu_{2} / \mu_{1}$ and $R / h . \sigma_{\max }$ is the maximum compressive stress in the contact area. a For $\mu_{2} / \mu_{1}=7$; b for $\mu_{2} / \mu_{1}=1 / 7$

case, a contrary phenomenon similar to the flat punch case can be found, i.e., the contact pressure distributes more uniformly for a decreasing layer thickness as shown in Fig. 5b. An interesting phenomenon is that either for the stiffer substrate case or a softer substrate one the effect of the layer thickness on the stress distributions almost vanishes when the thickness attains some relatively large value with a fixed contact width. This also proves the reasonability that one can use a finite-scale substrate to represent a half-space in some numerical simulations.

Consider the effect of friction. Figures $6 \mathrm{a}-\mathrm{d}$ show the interfacial stress distributions for both flat and cylindrical stamp cases. Similar results can be found in the frictional contact problem to the frictionless one. The friction coefficient does not show significant effects on the magnitude of the contact pressure as shown in Fig. 6a, b, but makes the contact pressure asymmetric as shown in Fig. 6c, d for flat punch and cylindrical punch cases, respectively. From Fig. 6a, b, the degree of asymmetry becomes larger with an increase of friction coefficient $\eta$ for a fixed ratio of stiffness $\mu_{2} / \mu_{1}$. All the phenomena are achieved under the assumption that no heat generates when a punch is sliding on the substrate.

\section{Conclusion}

In this paper, the contact behavior between a rigid punch and a homogeneous half-space coated with a linear graded layer is analyzed. It is found that the stress singularity depends only on the frictional coefficient and the surface Poisson's ratio and is independent of all the other material constants and length scales. The contact pressure concentrates near the edges of the flat punch, and the stress intensity factor at the trailing edge is larger than that at the leading one. When the frictional coefficient tends to zero, both singularities tend to the classical one, i.e., $-1 / 2$. The graded variation law and the thickness of the surface layer will show significant effects on the stress intensity factors and distributions of the contact pressure. Less stress concentration is produced 

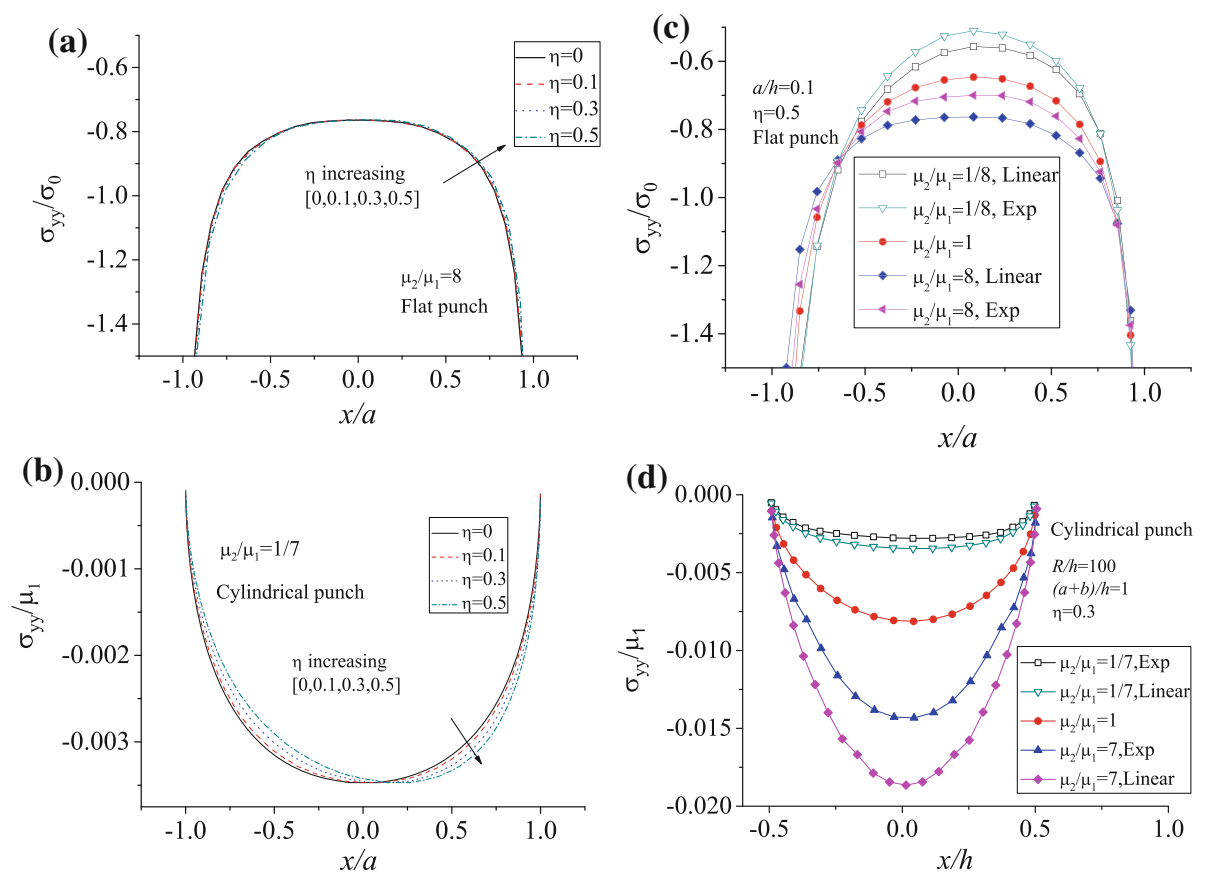

Fig. 6 The effects of the frictional contact on the contact pressure. a Effects of the friction coefficient $\eta$ on the contact pressure in a flat punch case; $\mathbf{b}$ effects of the friction coefficient $\eta$ on the contact pressure in a cylindrical punch case. $\mathbf{c}$ Distributions of contact pressure in the contact region for frictional contact models of a rigid flat punch and a linear graded-layered half-space or an exponential graded-layered one, with different values of $\mu_{2} / \mu_{1}$, and fixed $a / h=0.1, \eta=0.5 ; \mathbf{d}$ distributions of contact pressure in the contact region for frictional contact models of a rigid cylindrical punch and a linear graded-layered half-space or an exponential graded-layered one, with different values of $\mu_{2} / \mu_{1}$, and fixed $(a+b) / h, R / h, \eta=0.3$

near the flat punch edges when the surface layer obeys a linear variation law than in the case with an exponential graded law. There exists a limitation of the graded layer thickness, beyond which the influence of the thickness is weakened very quickly. All the results should be useful in interpreting experimental observations and potentially for the coating design of advanced engineering materials.

Acknowledgments The work reported here is supported by NSFC through Grants \#10972220, \#10732050, and \#11125211.

\section{References}

1. Ke, L.L., Wang, Y.S.: Two-dimensional sliding frictional contact of functionally graded materials. Eur. J. Mech. A-Solids 26, 171-188 (2007)

2. Hogmark, S., Jacobson, S., Larsson, M.: Design and evaluation of tribological coatings. Wear 246, 20-33 (2000)

3. Dag, S., Erdogan, F.: A surface crack in a graded medium loaded by a sliding rigid stamp. Eng. Fract. Mech. 69, 1729$1751(2002)$

4. Choi, H.J., Paulino, G.H.: Interfacial cracking in a graded coating/substrate system loaded by a frictional sliding flat punch. Proc. R. Soc. A-Math. Phys. Eng. Sci. 466, 853-880 (2010)

5. Ke, L.-L., Wang, Y.-S., Yang, J., Kitipornchai, S.: Sliding frictional contact analysis of functionally graded piezoelectric layered half-plane. Acta Mech. 209, 249-268 (2010)

6. Yao, S.H., Su, Y.L., Kao, W.H., Cheng, K.W.: A wear-resistant coating-oxidized graded multilayer TiN/W coating. Mater. Lett. 64, 99-101 (2010)

7. Zhou, Y., Li, X., Yu, D.: A partially insulated interface crack between a graded orthotropic coating and a homogeneous orthotropic substrate under heat flux supply. Int. J. Solids Struct. 47, 768-778 (2010)

8. Guler, M.A., Erdogan, F.: Contact mechanics of two deformable elastic solids with graded coatings. Mech. Mater. 38, 633-647 (2006)

9. Guler, M.A., Erdogan, F.: Contact mechanics of graded coatings. Int. J. Solids Struct. 41, 3865-3889 (2004)

10. Pender, D.C., Padture, N.P., Giannakopoulos, A.E., Suresh, S.: Gradients in elastic modulus for improved contact-damage resistance. Part I: The silicon nitride-oxynitride glass system. Acta Mater. 49, 3255-3262 (2001)

11. Pender, D.C., Thompson, S.C., Padture, N.P., Giannakopoulos, A.E., Suresh, S.: Gradients in elastic modulus for improved contact-damage resistance. Part II: The silicon nitride-silicon carbide system. Acta Mater. 49, 3263-3268 (2001)

12. Chen, W.Q.: On piezoelastic contact problem for a smooth punch. Int. J. Solids Struct. 37, 2331-2340 (2000) 
13. Ramaswamy, P., Seetharamu, S., Varma, K.B.R., Rao, K.J.: $\mathrm{Al}_{2} \mathrm{O}_{3}-\mathrm{ZrO}_{2}$ composite coatings for thermal-barrier applications. Compos. Sci. Technol. 57, 81-89 (1997)

14. Choi, H.J., Paulino, G.H.: Thermoelastic contact mechanics for a flat punch sliding over a graded coating/substrate system with frictional heat generation. J. Mech. Phys. Solids 56, 1673-1692 (2008)

15. He, L.H., Swain, M.V.: Enamel—a functionally graded natural coating. J. Dent. 37, 596-603 (2009)

16. Kashtalyan, M., Menshykova, M.: Effect of a functionally graded interlayer on three-dimensional elastic deformation of coated plates subjected to transverse loading. Compos. Struct. 89, 167-176 (2009)

17. Gorokovsky, V.I., Bowman, C., Gannon, P.E., VanVorous, D., Voevodin, A.A., Muratore, C., Kang, Y.S., Hu, J.J.: Deposition and characterization of hybrid filtered arc/magnetron multilayer nanocomposite cermet coatings for advanced tribological applications. Wear 265, 741-755 (2008)

18. Suresh, S., Olsson, M., Giannakopoulos, A.E., Padture, N.P., Jitcharoen, J.: Engineering the resistance to sliding-contact damage through controlled gradients in elastic properties at contact surfaces. Acta Mater. 47, 3915-3926 (1999)

19. Suresh, S.: Graded materials for resistance to contact deformation and damage. Science 292, 2447-2451 (2001)

20. Giannakopoulos, A.E., Suresh, S.: Indentation of solids with gradients in elastic properties. 1. Point force. Int. J. Solids Struct. 34, 2357-2392 (1997)

21. Giannakopoulos, A.E., Suresh, S.: Indentation of solids with gradients in elastic properties. 2. Axisymmetric indentors. Int. J. Solids Struct. 34, 2393-2428 (1997)

22. Chen, S.H., Yan, C., Zhang, P., Gao, H.J.: Mechanics of adhesive contact on a power-law graded elastic half-space. J. Mech. Phys. Solids 57, 1437-1448 (2009)

23. Chen, S.H., Yan, C., Soh, A.: Adhesive behavior of two-dimensional power-law graded materials. Int. J. Solids Struct. 46, 3398-3404 (2009)

24. Chen, S.H., Chen, P.J.: Nanoadhesion of a power-law graded elastic material. Chin. Phys. Lett. 27, 108102-1-3 (2010)

25. Dag, S., Guler, M.A., Yildirim, B., Cihan Ozatag, A.: Sliding frictional contact between a rigid punch and a laterally graded elastic medium. Int. J. Solids Struct. 46, 4038-4053 (2009)

26. Guler, M.A., Erdogan, F.: The frictional sliding contact problems of rigid parabolic and cylindrical stamps on graded coatings. Int. J. Mech. Sci. 49, 161-182 (2007)

27. Guler, M.A.: Mechanical modeling of thin films and cover plates bonded to graded substrates. J. Appl. Mech.-Trans. ASME 75, 051105 (2008)

28. Ke, L.L., Wang, Y.S.: Two-dimensional contact mechanics of functionally graded materials with arbitrary spatial variations of material properties. Int. J. Solids Struct. 43, 5779-5798 (2006)

29. Yang, J., Ke, L.L.: Two-dimensional contact problem for a coating-graded layer-substrate structure under a rigid cylindrical punch. Int. J. Mech. Sci. 50, 985-994 (2008)

30. Giannakopoulos, A.E., Pallot, P.: Two-dimensional contact analysis of elastic graded materials. J. Mech. Phys. Solids 48, 1597-1631 (2000)

31. Slater, L.J.: Confluent Hypergeometric Functions. Cambridge University Press, London (1960)

32. Krenk, S.: Quadrature formulas for singular integral equations of 1st and 2nd kind. Q. Appl. Math. 33, 225-232 (1975)

33. Muskhelishvili, N.L.: Singular Integral Equations. P. Noordhoff, Groningen (1953) 